Journal of Universal Mathematics

Vol.3 No.1 PP.59-65 (2020)

ISSN-2618-5660

\title{
ON GENERATED GROUPS
}

\author{
S.DEMIRALP AND G. HACAT
}

\begin{abstract}
In this paper we define a binary operation $\stackrel{\alpha}{*}$ on $Y$, called as generated operation. We have shown the basic features of this binary operation. The aim of this study is to define and investigate the properties of generated new semi groups and generated groups.
\end{abstract}

\section{Introduction and Preliminaries}

Let $G$ be a set and $\alpha: G \times G \rightarrow G$ a binary operation that maps each ordered pair $(x, y)$ of $G$ to an element $\alpha(x, y)$ of $G$. The pair $(G, \alpha)$ is called a groupoid. The mapping $\alpha$ is called a product of $(G, \alpha)$. The element $x y(=\alpha(x, y))$ is the product of $x$ and $y$ in $G$. We also use other notations for the product, when this is natural or otherwise convenient. In particular, the following symbols may be used: $\cdot,+, \star, \diamond, \oplus, \otimes$. If we do not explicitly mention the (notation for the) product, we choose the dot: $x \cdot y$. A groupoid $G$ is a semi group, if the operation $\alpha$ is associative: for all $x, y, z \in G, x \cdot(y \cdot z)=(x \cdot y) \cdot z$. That is, $\alpha(x, \alpha(y, z))=\alpha(\alpha(x, y), z)$ using the unfriendly notation. This means that the order in which the operation $\alpha$ is carried out is irrelevant, and therefore we may write

$$
\left.x_{1} x_{2} \ldots x_{n}=x_{1} \cdot\left(x_{2} \cdot\left(\cdots x_{n}\right)\right) \ldots\right) .
$$

Of course, the order of the operands $x_{1} x_{2} \ldots x_{n}$ (where we can have repeated elements, $x_{i}=x_{j}$ ), is important.

Generalized groups as an algebraic structure were deduced from a geometrical problem in 1998[3]. We recall that a group is a non-empty set $\mathrm{G}$ admitting an operation called multiplication, which satisfies the set of conditions given below:

i) $(x y) z=x(y z)$ for all $x, y, z \in G$,

ii) for each $x \in G$ there exists a unique $z \in G$ such that $x z=z x=x$ (we denote $z$ by $e(x))$,

iii) for each $x \in G$ there exists $y \in G$ such that $x y=y x=e(x)$.

\section{Theorem 1.1.}

i) For each $x \in G$ there exists a unique $x^{-1} \in G$.

ii) Let $G$ be a group and $a b=b a$ for all $a, b$ in $G$. Then $G$ is a group.

In this paper we define a binary operation on $Y$, called as generated operation. We have shown the basic features of this binary operation. The aim of this study is

Key words and phrases. Semigroups,groups,generated semigroups,generated groups. 
to define and investigate the properties of generated new semigroups and generated groups.

\section{The Construction}

Definition 2.1. Let $\emptyset \neq X$ be set with a binary operation $*$ and $\alpha: X \rightarrow Y$ be a bijective map. Then we can define a binary operation $*$ on $Y$, called as generated operation by $*$, such that, for all $y_{1}, y_{2} \in Y, y_{1} * y_{2}=\alpha\left[\alpha^{-1}\left(y_{1}\right) * \alpha^{-1}\left(y_{2}\right)\right]$.

Example 2.2. Let $X$ be an increasing sequence of different finite sets and let $s\left(A_{n}\right)$ is number of elements of set $A_{n}$. Let define a map $\alpha$ from $(X, \cup)$ onto a subset $\mathbb{N}^{\alpha}$ of $\mathbb{N}$ such that $\alpha\left(A_{n}\right)=s\left(A_{n}\right)$. Let $s\left(A_{n}\right)=n, s\left(A_{m}\right)=m$ and $n<m$. Then for all $n, m \in \mathbb{N}^{\alpha}$,

$$
\begin{aligned}
n \cup \stackrel{\alpha}{\cup} m & =\alpha\left(\alpha^{-1}(n) \cup \alpha^{-1}(m)\right) \\
& =\alpha\left(A_{n} \cup A_{m}\right) \\
& =\alpha\left(A_{m}\right) \\
& =s\left(A_{m}\right)=m .
\end{aligned}
$$

Theorem 2.3. If $(X, *)$ is a semigroup then $(Y, \stackrel{\alpha}{*})$ is semigroup.

Proof. It is clear that $\left(y_{1} \stackrel{\alpha}{*} y_{2}\right) \in Y$, for all $y_{1}, y_{2} \in Y$. Now let prove associativity. For all $y_{1}, y_{2}, y_{3} \in Y$,

$$
\begin{aligned}
y_{1} *\left(y_{2} * y_{3}\right) & =\alpha\left[\alpha^{-1}\left(y_{1}\right) * \alpha^{-1}\left(y_{2} * y_{3}\right)\right] \\
& =\alpha\left[\alpha^{-1}\left(y_{1}\right) * \alpha^{-1}\left(\alpha\left(\alpha^{-1}\left(y_{2}\right) * \alpha^{-1}\left(y_{3}\right)\right)\right)\right] \\
& =\alpha\left[\alpha^{-1}\left(y_{1}\right) *\left(\alpha^{-1}\left(y_{2}\right) * \alpha^{-1}\left(y_{3}\right)\right)\right] \\
& =\alpha\left[\left(\alpha^{-1}\left(y_{1}\right) * \alpha^{-1}\left(y_{2}\right)\right) * \alpha^{-1}\left(y_{3}\right)\right] \\
& =\alpha\left[\alpha^{-1}\left(y_{1} * y_{2}\right) * \alpha^{-1}\left(y_{3}\right)\right] \\
& =\left(y_{1} * y_{2}\right) * y_{3} .
\end{aligned}
$$

$(Y, \stackrel{\alpha}{*})$ is called as generated semigroup by $X$.

Theorem 2.4. If $(X, *)$ is a commutative semigroup, then $(Y, \underset{*}{*})$ is commutative.

Proof. It is trivial.

Theorem 2.5. Let $(X, *)$ be a semigroup. Then $e$ is the identity element of $(X, *)$ if and only if $\alpha(e)$ is identity for $(Y, \stackrel{\alpha}{*})$.

Proof. Let $e$ be the identity element and $\alpha(e)=e^{\prime}$. Then for all $y \in Y$,

$$
\begin{aligned}
y^{\alpha} e^{\prime} & =\alpha\left(\alpha^{-1}(y) * \alpha^{-1}\left(e^{\prime}\right)\right) \\
& =\alpha\left(\alpha^{-1}(y) * e\right) \\
& =\alpha\left(\alpha^{-1}(y)\right)=y .
\end{aligned}
$$

Similarly $e^{\prime} \stackrel{\alpha}{*} y=y$. Therefore $e^{\prime}$ is the identity element of $(Y, \stackrel{\alpha}{*})$. 
Let $\alpha(e)$ be identity element for $(Y, \stackrel{\alpha}{*})$. Then $y *{ }_{*}^{\prime} e^{\prime}=y$. Therefore for all $y \in Y$,

$$
\alpha\left(\alpha^{-1}(y) * \alpha^{-1}\left(e^{\prime}\right)\right)=y \Rightarrow \alpha\left(x * \alpha^{-1}\left(e^{\prime}\right)\right)=y .
$$

Similarly $\alpha^{-1}\left(e^{\prime}\right) * x=x$. So $\alpha(e)$ is identity element for $(X, *)$.

Theorem 2.6. If $e^{\prime}$ is the identity element of $(Y, \stackrel{\alpha}{*})$, then $e^{\prime}$ is uniq.

Proof. Consider $e_{1}^{\prime}$ and $e_{2}^{\prime}$ are two diffirent identity element for $\stackrel{\alpha}{*}$. Then for all $y \in Y, y^{*} e_{1}^{\prime}=y \stackrel{\alpha}{*} e_{2}^{\prime}$. Therefore,

$$
\begin{aligned}
\alpha\left(\alpha^{-1}(y) * \alpha^{-1}\left(e_{1}^{\prime}\right)\right) & =\alpha\left(\alpha^{-1}(y) * \alpha^{-1}\left(e_{2}^{\prime}\right)\right) \\
& \Rightarrow \alpha\left(\alpha^{-1}(y) * \alpha^{-1}\left(e_{1}^{\prime}\right)\right)=\alpha\left(\alpha^{-1}(y) * \alpha^{-1}\left(e_{2}^{\prime}\right)\right)=y \\
& \Rightarrow \alpha^{-1}(y) * \alpha^{-1}\left(e_{1}^{\prime}\right)=\alpha^{-1}(y) * \alpha^{-1}\left(e_{2}^{\prime}\right)=x \\
& \Rightarrow x * \alpha^{-1}\left(e_{1}^{\prime}\right)=x * \alpha^{-1}\left(e_{2}^{\prime}\right)=x .
\end{aligned}
$$

But this is a contradiction since the unit element of $(X, *)$ is unit.

Let for a binary operation $\oplus,(\oplus)^{n}(y)=\underbrace{y \oplus y \oplus \ldots \oplus y}_{n \text {-times }}$.

Theorem 2.7. Let $(X, *)$ be a semigroup. Then for all $y, y_{1}, y_{2} \in Y$,

(1) $\left(\begin{array}{l}\alpha \\ *\end{array}\right)^{n}(y)=\alpha\left((*)^{n}\left(\alpha^{-1}(y)\right)\right)$

(2) $\left(\begin{array}{l}\alpha \\ *\end{array}\right)^{m}\left(\left(\begin{array}{l}\alpha \\ *\end{array}\right)^{n}(y)\right)=\left(\begin{array}{l}\alpha \\ *\end{array}\right)^{n m}(y)$

(3) $\left(\begin{array}{l}\alpha \\ *\end{array}\right)^{n}(y){ }^{\alpha}\left(\begin{array}{l}\alpha \\ *\end{array}\right)^{m}(y)=\left(\begin{array}{l}\alpha \\ *\end{array}\right)^{m}(y){ }^{\alpha}\left(\begin{array}{l}\alpha \\ *\end{array}\right)^{m}(y)=\left(\begin{array}{l}\alpha \\ *\end{array}\right)^{n+m}(y)$

(4) If $(X, *)$ is commutative, then $\left(\begin{array}{l}\alpha \\ *\end{array}\right)^{n}\left(y_{1} * y_{2}\right)=\left(\begin{array}{l}\alpha \\ *\end{array}\right)^{n}\left(y_{1}\right) *\left(\begin{array}{l}\alpha \\ *\end{array}\right)^{n}\left(y_{2}\right)$.

Proof.

$$
\begin{aligned}
& \left(\begin{array}{l}
\alpha \\
*
\end{array}\right)^{n}(y)=\underbrace{y^{\alpha} y^{\alpha} \ldots * 2}_{n \text {-times }} \stackrel{\alpha}{*}
\end{aligned}
$$

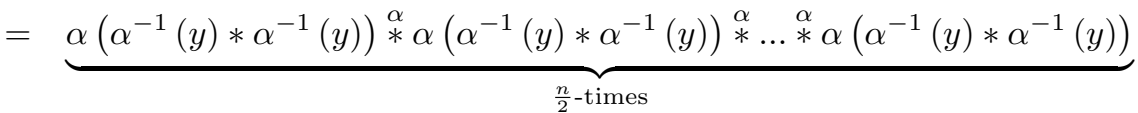

$$
\begin{aligned}
& =\alpha\left((*)^{2}\left(\alpha^{-1}(y)\right)\right) \stackrel{\alpha}{*} \alpha\left((*)^{2}\left(\alpha^{-1}(y)\right)\right) \stackrel{\alpha}{*} \ldots * \underset{*}{*} \alpha\left((*)^{2}\left(\alpha^{-1}(y)\right)\right) \\
& =\left(\alpha\left(\alpha^{-1}\left(\alpha\left((*)^{2}\left(\alpha^{-1}(y)\right)\right)\right)\right) * \alpha^{-1}\left(\alpha\left((*)^{2}\left(\alpha^{-1}(y)\right)\right)\right)\right) * \ldots \\
& =\underbrace{\ldots * *\left(\alpha\left(\alpha^{-1}\left(\alpha\left((*)^{2}\left(\alpha^{-1}(y)\right)\right)\right)\right) * \alpha^{-1}\left(\alpha\left((*)^{2}\left(\alpha^{-1}(y)\right)\right)\right)\right) .}_{\frac{n}{4} \text {-times }} \\
& =\alpha\left((*)^{4}\left(\alpha^{-1}(y)\right)\right) \stackrel{\alpha}{*} \alpha\left((*)^{4}\left(\alpha^{-1}(y)\right)\right) \stackrel{\alpha}{*} \ldots{ }^{*} \alpha\left((*)^{4}\left(\alpha^{-1}(y)\right)\right) \\
& =\alpha\left((*)^{n}\left(\alpha^{-1}(y)\right)\right) \text {. }
\end{aligned}
$$


(2)

$$
\begin{aligned}
\left(\begin{array}{l}
\alpha \\
*
\end{array}\right)^{m}\left(\left(\begin{array}{l}
\alpha \\
*
\end{array}\right)^{n}(y)\right) & =\alpha\left((*)^{m}\left(\alpha^{-1}\left(\left(\begin{array}{l}
\alpha \\
*
\end{array}\right)^{n}(y)\right)\right)\right) \\
& =\alpha\left((*)^{m}\left(\alpha^{-1}\left(\alpha(*)^{n}(y)\right)\right)\right) \\
& =\alpha\left((*)^{m}\left((*)^{n}(y)\right)\right) \\
& =\alpha\left((*)^{n m}(y)\right) .
\end{aligned}
$$

(3)

$$
\begin{aligned}
\left(\begin{array}{l}
\alpha \\
*
\end{array}\right)^{n}(y){ }^{\alpha}\left(\begin{array}{l}
\alpha \\
*
\end{array}\right)^{m}(y) & =\alpha\left((*)^{n}\left(\alpha^{-1}(y)\right)\right) \stackrel{\alpha}{*} \alpha\left((*)^{m}\left(\alpha^{-1}(y)\right)\right) \\
& =\alpha\left[\alpha^{-1}\left(\alpha\left((*)^{n}\left(\alpha^{-1}(y)\right)\right)\right) * \alpha^{-1}\left(\alpha\left((*)^{m}\left(\alpha^{-1}(y)\right)\right)\right)\right] \\
& =\alpha\left[(*)^{n}\left(\alpha^{-1}(y)\right) *(*)^{m}\left(\alpha^{-1}(y)\right)\right] \\
& =\alpha(*)^{n+m}\left(\alpha^{-1}(y)\right) .
\end{aligned}
$$

(4)

$$
\begin{aligned}
& \left(\begin{array}{l}
\alpha \\
*
\end{array}\right)^{n}\left(y_{1} * y_{2}\right)=\alpha\left((*)^{n}\left(\alpha^{-1}\left(y_{1} * y_{2}^{*}\right)\right)\right) \\
& =\alpha\left((*)^{n}\left(\alpha^{-1}\left(\alpha\left(\alpha^{-1}\left(y_{1}\right) * \alpha^{-1}\left(y_{2}\right)\right)\right)\right)\right) \\
& =\alpha\left((*)^{n}\left(\alpha^{-1}\left(y_{1}\right) * \alpha^{-1}\left(y_{2}\right)\right)\right) \\
& \left(\begin{array}{l}
\alpha \\
*
\end{array}\right)^{n}\left(y_{1}\right) *\left(\begin{array}{l}
\alpha \\
*
\end{array}\right)^{n}\left(y_{2}\right)=\alpha\left((*)^{n}\left(\alpha^{-1}\left(y_{1}\right)\right)\right) \stackrel{\alpha}{*} \alpha\left((*)^{n}\left(\alpha^{-1}\left(y_{2}\right)\right)\right) \\
& =\alpha\left((*)^{n}\left(\alpha^{-1}\left(y_{1}\right)\right) *(*)^{n}\left(\alpha^{-1}\left(y_{2}\right)\right)\right) \\
& =\alpha\left((*)^{n}\left(\alpha^{-1}\left(y_{1}\right)\right) * \alpha^{-1}\left(y_{2}\right)\right) \text {. }
\end{aligned}
$$

Theorem 2.8. Let $(Y, *)$ be a generated semigroup by $X$. Then $X \times Y$ is a semigroup.

Proof. Let define $\bullet: X \times Y \times X \times Y \longrightarrow X \times Y$ as $\bullet=(* \times * *) \circ\left(1_{X} \times T \times 1_{Y}\right)$, where $T(x, y)=(y, x)$. Then

$$
\begin{aligned}
\bullet(x, \bullet(y, z)) & =\left(* \times *^{\alpha}\right) \circ\left(1_{S} \times T \times 1_{T}\right)\left(x,\left(\left(* \times *^{\alpha}\right) \circ\left(1_{S} \times T \times 1_{T}\right)\right)\left(\left(y_{1}, y_{2}\right),\left(z_{1}, z_{2}\right)\right)\right) \\
& =(* \times *) \circ\left(1_{S} \times T \times 1_{T}\right)\left(x,\left((* \times *)\left(\left(y_{1}, z_{1}\right),\left(y_{2}, z_{2}\right)\right)\right)\right) \\
& =(* \times *) \circ\left(1_{S} \times T \times 1_{T}\right)\left(\left(x_{1}, x_{2}\right),\left(\left(y_{1} * z_{1}\right),\left(y_{2} * z_{2}\right)\right)\right. \\
& =(* \times *)\left(\left(x_{1},\left(y_{1} * z_{1}\right)\right),\left(x_{2},\left(y_{2} *^{\alpha} z_{2}\right)\right)\right) \\
& =(* \times *)\left(\left(x_{1},\left(y_{1} * z_{1}\right)\right),\left(x_{2}, \alpha\left(\alpha^{-1}\left(y_{2}\right) * \alpha^{-1}\left(z_{2}\right)\right)\right)\right) \\
& =\left(\left(x_{1} *\left(y_{1} * z_{1}\right)\right), *\left(x_{2}, \alpha\left(\alpha^{-1}\left(y_{2}\right) * \alpha^{-1}\left(z_{2}\right)\right)\right)\right) \\
& =\left(\left(x_{1} *\left(y_{1} * z_{1}\right)\right), \alpha\left(\alpha^{-1}\left(x_{2}\right), \alpha^{-1}\left(\alpha\left(\alpha^{-1}\left(y_{2}\right) * \alpha^{-1}\left(z_{2}\right)\right)\right)\right)\right) \\
& =\left(\left(x_{1} *\left(y_{1} * z_{1}\right)\right), \alpha\left(\alpha^{-1}\left(x_{2}\right),\left(\alpha^{-1}\left(y_{2}\right) * \alpha^{-1}\left(z_{2}\right)\right)\right)\right) .
\end{aligned}
$$




$$
\begin{aligned}
\bullet \bullet(x, y), z) & =(* \times *) \circ\left(1_{S} \times T \times 1_{T}\right)\left(\left((* \times *) \circ\left(1_{S} \times T \times 1_{T}\right)\right)\left(\left(x_{1}, x_{2}\right),\left(y_{1}, y_{2}\right)\right),\left(z_{1}, z_{2}\right)\right) \\
& =(* \times *) \circ\left(1_{S} \times T \times 1_{T}\right)\left((* \times *)\left(\left(x_{1}, y_{1}\right),\left(x_{2}, y_{2}\right)\right),\left(z_{1}, z_{2}\right)\right) \\
& =(* \times *) \circ\left(1_{S} \times T \times 1_{T}\right)\left(\left(\left(x_{1} * y_{1}\right), \alpha\left(\alpha^{-1}\left(x_{2}\right) * \alpha^{-1}\left(y_{2}\right)\right)\right),\left(z_{1}, z_{2}\right)\right) \\
& =(* \times *)\left(\left(\left(x_{1} * y_{1}\right), z_{1}\right),\left(\alpha\left(\alpha^{-1}\left(x_{2}\right) * \alpha^{-1}\left(y_{2}\right)\right), z_{2}\right)\right) \\
& =\left(\left(\left(x_{1} * y_{1}\right) * z_{1}\right), \alpha\left(\alpha^{-1}\left(\alpha\left(\alpha^{-1}\left(x_{2}\right) * \alpha^{-1}\left(y_{2}\right)\right)\right)\right), \alpha^{-1}\left(z_{2}\right)\right) \\
& =\left(\left(\left(x_{1} * y_{1}\right) * z_{1}\right), \alpha\left(\alpha^{-1}\left(x_{2}\right),\left(\alpha^{-1}\left(y_{2}\right) * \alpha^{-1}\left(z_{2}\right)\right)\right)\right) .
\end{aligned}
$$

Therefore $\bullet$ is associative.

Remark 2.9. If $e$ is the identity element of $(X, *)$, then $(*)^{n}(\alpha(e))=\alpha(e)$.

Remark 2.10. If $(X, *)$ a monoid, then $(Y, *)$ is monoid.

Theorem 2.11. Let $(X, *)$ has a zero element $z$. Then $\alpha(z)$ is zero element for $(Y, *)$

Proof. Let $z$ be a zero element of $(X, *)$ Then for all $x \in X, x * z=z * x=z$. Let $\alpha(x)=y$. Then

$$
\begin{aligned}
& \stackrel{\alpha}{*} \alpha(z)=\alpha\left(\alpha^{-1}(y) * z\right)=\alpha(x * z)=\alpha(z) \\
& \alpha(z) \stackrel{\alpha}{*} y=\alpha\left(z * \alpha^{-1}(y)\right)=\alpha(z * x)=\alpha(z) .
\end{aligned}
$$

Let $z^{\prime}$ be a zero element for $\left(Y, \mu^{\alpha}\right)$ and $\alpha^{-1}\left(z^{\prime}\right)=z$.

$$
\begin{aligned}
y^{\alpha} z^{\prime} & =\alpha\left(\alpha^{-1}(y) * \alpha^{-1}\left(z^{\prime}\right)\right)=z^{\prime} \\
& \Rightarrow\left(\alpha^{-1}(y) * \alpha^{-1}\left(z^{\prime}\right)\right)=z
\end{aligned}
$$

and

$$
z^{\prime} * \underset{\alpha}{*}=\alpha\left(\alpha^{-1}\left(z^{\prime}\right) * \alpha^{-1}(y)\right)=z^{\prime} \Rightarrow\left(\alpha^{-1}\left(z^{\prime}\right) * \alpha^{-1}(y)\right)=z .
$$

So $z$ is zero element for $(X, *)$.

Theorem 2.12. Let $(X, *)$ be a group, then $(Y, \stackrel{\alpha}{*})$ is a group, called as generated group by $X$.

Proof. It is clear that $(Y, \stackrel{\alpha}{*})$ is a semi group with the identity $\alpha(e)=e^{\prime}$. Let show that for any $y \in Y$, there exist a $y^{-1} \notin Y$ such that $y \stackrel{\alpha}{*} y^{-1}=y^{-1} \stackrel{\alpha}{*} y=e^{\prime}$.

Let $\alpha(x)=y$ and $\alpha\left(x^{-1}\right)=y^{-1}$. Then

$$
\begin{aligned}
y^{\alpha} y^{-1} & =\alpha\left(\alpha^{-1}(y) * \alpha^{-1}\left(y^{-1}\right)\right) \\
& =\alpha\left(x * x^{-1}\right) \\
& =\alpha(e)=e^{\prime} .
\end{aligned}
$$

Similarly $y^{-1} \stackrel{\alpha}{*}=e^{\prime}$.

If $(X, *)$ is a group and $\alpha: X \rightarrow Y$ is a bijective map, then it is clear that $\alpha$ is a group homomorphism.

Theorem 2.13. Let $(X, *)$ be an abelian group, then $\left(Y,{ }_{*}^{\alpha}\right)$ is an abelian group.

Proof. For all $y_{1}, y_{2} \in Y, y_{1} \stackrel{\alpha}{*} y_{2}=\alpha\left(\alpha^{-1}\left(y_{1}\right) * \alpha^{-1}\left(y_{2}\right)\right)=y_{2} \stackrel{\alpha}{*} y_{1}$. 
Theorem 2.14. Let $(Y, *)$ be a generated group by $X$. Then,

i) $y^{\alpha} * y=y \Rightarrow y=\alpha(e)$

ii) $y_{1} \stackrel{\alpha}{*} y_{2}=y_{1} \stackrel{\alpha}{*} y_{3} \Rightarrow y_{2}=y_{3}$

iii) $\left(y^{-1}\right)^{-1}=y$ for all $y \in X$.

Proof. $\quad$ i Let $y^{*} \underset{y}{*}=y$. Then,

$$
y^{-1} \stackrel{\alpha}{*}(y \stackrel{\alpha}{*} y)=y^{-1} \stackrel{\alpha}{*} y .
$$

Since $y^{-1} \stackrel{\alpha}{*}(y \stackrel{\alpha}{*} y)=\left(y^{-1} \stackrel{\alpha}{*} y\right) \stackrel{\alpha}{*} y, e^{*} * y=y$.Hence $y=e$.

ii Let $y_{1} * y_{2}=y_{1} *{ }^{*} y_{3}$ for all $y_{1}, y_{2}, y_{3} \in X$. Then,

$$
\begin{aligned}
y_{1}^{-1} \stackrel{\alpha}{*}\left(y_{1} * y_{2}\right) & =y_{1}^{-1} \stackrel{\alpha}{*}\left(y_{1} * y_{3}\right) \\
\left(y_{1}^{-1} \stackrel{\alpha}{*} y_{1}\right) \stackrel{\alpha}{*} y_{2} & =\left(y_{1}^{-1} \stackrel{\alpha}{*} y_{1}\right) * y_{3} \\
e * y_{2} & =e * y_{3} \\
y_{2} & =y_{3} .
\end{aligned}
$$

iii If $y \in X$ is the inverse of $y^{-1}$,then

$$
y^{*}{ }^{-1}=y^{-1} \stackrel{\alpha}{*} y=e .
$$

Since $\left(y^{-1}\right)^{-1}$ is the inverse of $y^{-1}, y^{-1} \stackrel{\alpha}{*}\left(y^{-1}\right)^{-1}=e=y^{-1} \stackrel{\alpha}{*} y$. Therefore by iii, $\left(y^{-1}\right)^{-1}=y$.

Theorem 2.15. Let $(Y, \stackrel{\alpha}{*})$ be a generated group by $X$. Then

$$
\left(y_{1} \stackrel{\alpha}{*} y_{2}\right)^{-1}=y_{2}^{-1} \stackrel{\alpha}{*} y_{1}^{-1} .
$$

Proof.

$$
\begin{aligned}
\left(y_{1} * y_{2}\right) *\left(y_{2}^{-1} \stackrel{\alpha}{*} y_{1}^{-1}\right) & =y_{1} *\left(\left(y_{2} * y_{2}^{-1}\right) * y_{1}^{-1}\right) \\
& =y_{1} *\left(e^{\alpha} y_{1}^{-1}\right) \\
& =y_{1} * y_{1}^{-1} \\
& =e .
\end{aligned}
$$

Similarly,

$$
\begin{aligned}
& \left(y_{2}^{-1} \stackrel{\alpha}{*} y_{1}^{-1}\right) * \underset{*}{*}\left(y_{1} * y_{2}\right)=e \\
& \left(y_{1} * \underset{y_{2}}{\stackrel{\alpha}{*}}\right)^{\alpha}\left(y_{2}^{-1} \stackrel{\alpha}{*} y_{1}^{-1}\right)=\left(y_{2}^{-1} \stackrel{\alpha}{*} y_{1}^{-1}\right) *\left(y_{1}^{*} * y_{2}\right)=e \\
& \left(y_{1} * y_{2}\right)^{-1}=y_{2}^{-1} \stackrel{\alpha}{*} y_{1}^{-1} \text {. }
\end{aligned}
$$

Theorem 2.16. Let $\left(Y,{ }_{*}^{\alpha}\right)$ be a generated group by $X$. Then $X \times Y$ is a group. 
Proof. By the theorem $2.8(X \times Y, \bullet)$ is a semigroup. Let show that $e^{\prime}=(e, \alpha(e))$ is the identity element of $(X \times Y, \bullet)$ where $e$ is the identity element of $(X, *)$.

$$
\left(\left(x_{1}, x_{2}\right) \bullet(e, \alpha(e))\right)=\left(\left(x_{1} * e\right),\left(x_{2}, \alpha(e)\right)\right)=\left(x_{1}, x_{2}\right) .
$$

Now let show that for any $(x, y) \in X \times Y,\left(x^{-1}, \alpha\left(\left(\alpha^{-1}(y)\right)^{-1}\right)\right)$ is the inverse element of $(x, y)$.

$$
\begin{aligned}
(x, y) \bullet\left(x^{-1}, \alpha\left(\left(\alpha^{-1}(y)\right)^{-1}\right)\right) & =\left(x * x^{-1}, y * \alpha\left(\left(\alpha^{-1}(y)\right)^{-1}\right)\right) \\
& =\left(e, \alpha\left(\alpha^{-1}(y) * \alpha^{-1}\left(\alpha\left(\left(\alpha^{-1}(y)\right)^{-1}\right)\right)\right)\right) \\
& =\left(e, \alpha\left(\alpha^{-1}(y) *\left(\alpha^{-1}(y)\right)^{-1}\right)\right) \\
& =(e, \alpha(e)) .
\end{aligned}
$$

\section{REFERENCES}

[1] D. Tascı,Soyut Cebir,Alp Yayınevi, (2007).

[2] J.M. Howie, Fundamental of Semigroup Theory, Oxford University Press, 1995.

[3] M.R. Molaei , Generalized Groups, International Conference on Algebra, October 14-17, Romania, 1998, Buletinul Institului Polithnic Din Iasi, Tomul XLV (XLIX) , 21-24,(1999).

[4] T.W. Judson,Abstract Algebra Theory and Applications,Stephen F. Austin State University (2010).

[5] Z. Hedrlin and A. Pultr, Relations (Graphs) with Given Finitely Generated Semigroups, Springer Book, Volume 68, Issue 3, pp 213-217, (1964).

Kastamonu University, Mathematics Department, 37000, Kastamonu, Turkey

Current address: Kastamonu University, Mathematics Department, 37000, Kastamonu, Turkey

Email address: sdemiralp@kastamonu.edu.tr

Kastamonu University, Mathematics Department, 37000, Kastamonu, Turkey

Email address: ghacat@ogr.kastamonu.edu.tr 